\title{
MicroRNA-758 acts as a tumor inhibitor in colorectal cancer through targeting PAX6 and regulating PI3K/AKT pathway
}

\author{
XINLI ZHANG ${ }^{1}$, JUMEI XU ${ }^{2}$, HONGCHANG ZHANG $^{3}$, JILAN SUN $^{4}, \mathrm{NA} \mathrm{LI}^{5}$ and XIANYU HUANG ${ }^{6}$ \\ ${ }^{1}$ Medical Records Management Office, Qingdao West Coast New Area Central Hospital, Qingdao, Shandong 266555; \\ Departments of ${ }^{2}$ Hepatobiliary Gastrointestinal Surgery and ${ }^{3}$ Infectious Diseases; ${ }^{4}$ Sterilization Supply Room; \\ ${ }^{5}$ Department of Radiology, The People's Hospital of Zhangqiu Area, Jinan, Shandong 250200; ${ }^{6}$ Radiotherapy Center, \\ Qingdao Central Hospital, Qingdao University, Qingdao, Shandong 266042, P.R. China
}

Received August 12, 2019; Accepted February 6, 2020

DOI: $10.3892 / \mathrm{ol} .2020 .11516$

\begin{abstract}
Recently, a number of microRNAs (miRNAs) have been reported to play different roles in human cancers, including colorectal cancer (CRC). However, the specific role of miR-758 has not been clarified in CRC. Therefore, the aim of the present study was to explore the role of miR-758 in CRC. RT-qPCR and western blot analysis were used to quantify the expression of miR-758 and genes. The function of miR-758 in CRC was investigated using Transwell, CCK- 8 and luciferase reporter assays. According to the results, the downregulation of miR-758 expression was associated with aggressive behavior and poor prognosis in CRC patients. miR-758 was shown to restrain the cell viability and metastasis in CRC. In addition, it was confirmed that miR-758 directly targets PAX6 and inhibits CRC progression through targeting PAX6. The results also revealed that miR-758 blocked EMT and PI3K/AKT pathway in CRC. In conclusion, miR-758 acts as a tumor suppressor in CRC by downregulating PAX6.
\end{abstract}

\section{Introduction}

Colorectal cancer (CRC) is a common malignant tumor of the gastrointestinal tract. The incidence and mortality of the digestive system malignancies rank second only to gastric, esophageal and primary liver cancers (1). Moreover, CRC is prone to invade and metastasize, which is the main cause of death. Surgical treatment is still the first choice for the radical treatment of CRC. However, despite the rapid development of surgical techniques, the surgical cure rate and the 5-year survival rate of CRC patients have been hovering around $50 \%$ (2). The recurrence of cancer in liver, lung or peritoneal

Correspondence to: Dr Xianyu Huang, Radiotherapy Center, Qingdao Central Hospital, Qingdao University, 127 Siliu South Road, Qingdao, Shandong 266042, P.R. China

E-mail: huucuc53273@163.com

Key words: miR-758, colorectal cancer, proliferation, metastasis, PAX6, PI3K/AKT pathway is one of the causes of CRC treatment failure (3). Therefore, early diagnosis and timely treatment is the key to improving the prognosis of CRC patients.

MicroRNAs (miRNAs) are small, non-coding RNAs that regulate gene expression. It has been reported that miRNAs have important functions in cell-cell communication and have the potential to detect and monitor diseases, such as cancer (4). A number of miRNAs have been found to act as tumor suppressors or promoters in a number of cancers, including CRC. For example, miR-141-3p has been shown to restrain the cell growth and metastasis by targeting TRAF5 in CRC (5). Furthermore, miR-182 has been reported to promote cell proliferation, invasion and tumor growth in CRC via regulating DAB2IP (6). Recently, the abnormal expression of miR-758 was found in human cancers, except CRC. In particular, the overexpression of miR-758 was demonstrated to inhibit cell proliferation, migration, invasion and promote apoptosis in non-small cell lung cancer by negatively regulating HMGB (7). Meng et al also showed that miR-758 was downregulated in cervical cancer, and miR-758 overexpression promoted cell invasion by regulating the expression of MEPE (8). These results suggest that miR-758 can be stably expressed in human tissues. However, the specific role of miR-758 in CRC has not been clarified yet.

TargetScan database (http://www.targetscan.org) predicts that paired box 6 (PAX6) is a target of miR-758. PAX6 has been found to be a highly conserved transcription factor that plays a crucial role in the development of human cancers (9). For example, PAX6 overexpression has been shown to promote tumor growth and inhibit apoptosis in human retinoblastoma (10). PAX-6 has also been shown to promote breast cancer cell proliferation and tumorigenesis (11). In addition, Needhamsen et al have demonstrated that PAX6 expression was regulated by miR-7 (12). However, the interaction between miR-758 and PAX6 has not been reported in CRC. Furthermore, the PI3K/AKT pathway is well known to be an important regulator in human cancers. The PI3K/AKT pathway has been demonstrated to be involved in tumor growth including CRC. The PI3K/AKT signaling pathway is associated with dysregulation of miRNA and gene expression in CRC (13). For example, miR-532 has been shown to inhibit CRC progression by directly targeting IGF-1R and suppressing the PI3K/AKT 
signaling pathway (14). However, the way miR-758 regulates the PI3K/AKT signaling pathway in CRC is unclear.

In the present study, the molecular mechanism of miR-758/PAX6/PI3K/AKT pathway was explored and the function of miR-758 was investigated in CRC, aiming to open up a new avenue for the diagnosis and treatment of CRC.

\section{Materials and methods}

Clinical tissues. The study was performed on 84 fresh CRC tissues (colon and rectal cancers) confirmed by histopathology at the Qingdao West Coast New Area Central Hospital (Qingdao, China) from May 2017 to January 2019. All patients with CRC did not receive any treatment before surgery. The study was approved by the Institutional Ethics Committee of the Qingdao West Coast New Area Central Hospital. Patients who participated in this research had complete clinical data. Signed written informed consents were obtained from the patients and/or guardians.

Cell culture. Normal human intestinal epithelial cells HIEC-6 and CRC cell lines HCT-116 and SW620 were purchased from ATCC. The cells were incubated in DMEM (10\% FBS, $5 \% \mathrm{CO}_{2}, 37^{\circ} \mathrm{C}$ ) for subsequent experimentation.

Cell transfection. miR-758 mimics, miR-758 inhibitor, and PAX6 overexpression plasmid were purchased from Shanghai GenePharma Co., Ltd. HCT-116 cells were transfected using Lipofectamine ${ }^{\circledR} 2000$ (Invitrogen, Thermo Fisher Scientifc, Inc.), respectively. HCT-116 cells with human homologous sequences were used as control (miR-NC). According to the manufacturer's protocol, the cells were plated in 6-well plates (at 70-90\% confluence) and were transfected with 100 pmol miR-758 mimics, inhibitor and their control or $2.5 \mu \mathrm{g}$ PAX6 overexpression plasmid. After $6 \mathrm{~h}$ of incubation at $37^{\circ} \mathrm{C}$ with $5 \% \mathrm{CO}_{2}$, the transfection efficiency was detected and subsequent assays were performed.

$R N A$ isolation and RT-qPCR. Total RNA isolation was performed using TRIzol ${ }^{\circledR}$ reagent (Invitrogen; Thermo Fisher Scientific, Inc.). The cDNA solution was then obtained using a PrimeScript Reverse Transcription kit (Qiagen China Co., Ltd). The temperature conditions for reverse transcription were as follows: $37^{\circ} \mathrm{C}$ for $15 \mathrm{~min}$ and $85^{\circ} \mathrm{C}$ for $5 \mathrm{sec}$. RT-qPCR was performed using SYBR Green I (Takara Biotechnology Co., Ltd.) following the manufacturer's instructions. The thermocycling conditions for PCR amplification were as follows: $5 \mathrm{~min}$ at $95^{\circ} \mathrm{C}$, followed by 40 cycles of $95^{\circ} \mathrm{C}$ for $30 \mathrm{sec}$ and $60^{\circ} \mathrm{C}$ for $45 \mathrm{sec}$. U6 and GAPDH were used as the controls for miR-758 and PAX6, respectively. The expression of miR-758 and PAX6 was quantified using the $2^{-\Delta \Delta \mathrm{Cq}}$ method (15). Primers were designed as follows: miR-758 forward, 5'-ACA CTCCAGCTGGGTTTGTGACCTGGTCCA-3' and reverse, 5'-TGGTGTCGTGGAGTCG-3'; U6 forward, 5'-CGCTTC GGCAGCACATATACTA-3' and reverse, 5'-GCGAGCACA GAATTAATACGAC-3'; PAX6 forward, 5'-TCTTTGCTT GGGAAATCCG-3' and reverse, 5'-CTGCCCGTTCAACAT CCTTAG-3'; and GAPDH forward, 5'-GGCACTGAGAAG CGGGGCCG-3' and reverse, 5'-GAAGATGGTGATGGG ATTTC-3'.
Transwell assay. An $8.0-\mu \mathrm{m}$ pore polycarbonate membrane insert (Corning, Inc.) was used to determine the invasion and migration of CRC cells. The upper chamber was coated with Matrigel (BD Biosciences) for invasion assay. Next, the transfected HCT-116 cells $\left(3 \times 10^{3}\right.$ cells/well) were placed in the upper chamber. DMEM with 10\% FBS was added to the lower chamber. After $24 \mathrm{~h}$, the cells were fixed and stained with $0.5 \%$ crystal violet at room temperature for $30 \mathrm{~min}$. For cell migration assay, Matrigel was not required and the procedure followed was identical to that of the cell invasion assay. Observation and image capturing were performed using a light microscope (magnification, $\mathrm{x} 200$ ).

CCK-8 assay. The prepared HCT-116 cells were incubated in a 96-well plate for $24 \mathrm{~h}\left(\right.$ at $\left.37^{\circ} \mathrm{C}, 5 \% \mathrm{CO}_{2}\right)$. HCT-116 cells $\left(4 \times 10^{3} /\right.$ well) were then incubated for $24,48,72$ and $96 \mathrm{~h}$. Next, the cells were incubated for $4 \mathrm{~h}$ with $10 \mathrm{ml}$ of CCK-8 (Dojindo Molecular Technologies, Inc.) solution. The absorbance at $450 \mathrm{~nm}$ was observed with a microplate reader (Molecular Devices, LLC).

Western blot analysis. Protein samples were obtained using RIPA lysis buffer (Beyotime Institute of Biotechnology). Total protein was quantified using a bicinchoninic acid protein assay kit (Pierce; Thermo Fisher Scientific, Inc.) and $40 \mu \mathrm{g}$ protein/lane were separated via SDS-PAGE on a $10 \%$ gel. The separated proteins were transferred onto polyvinylidene fluoride membranes (Thermo Fisher Scientific, Inc.) and blocked for $3 \mathrm{~h}$ at room temperature with $5 \%$ non-fat milk in PBS (Thermo Fisher Scientific, Inc.) containing 0.1\% Tween-20 (Sigma-Aldrich; Merck KGaA). The membranes were then incubated with E-cadherin (rabbit monoclonal; dilution, 1:1,000; cat. no. ab1416; Abcam), N-cadherin (rabbit polyclonal; dilution, 1:1,000; cat. no. ab18203; Abcam), Bcl-2 (rabbit monoclonal; dilution, 1:1,000; cat. no. ab185002; Abcam), Bax (mouse monoclonal; dilution, 1:1,000; cat. no. ab77566; Abcam), PI3K (rabbit monoclonal; dilution, 1:1,000; cat. no. ab32089; Abcam), p-PI3K (rabbit monoclonal; dilution, 1:1,000; cat. no. ab154598; Abcam), AKT (rabbit polyclonal; dilution, 1:1,000; cat. no. ab8805; Abcam), p-AKT (rabbit monoclonal; dilution, 1:1,000; cat. no. ab81283; Abcam) and GAPDH (rabbit monoclonal; dilution, 1:1,000; cat. no. ab181602; Abcam) primary antibodies overnight at $4^{\circ} \mathrm{C}$. After washing, the membranes were incubated with horseradish peroxidase-conjugated secondary antibodies (dilution, 1:5,000; cat. no. ab7090; Abcam) for $1 \mathrm{~h}$ at room temperature. Protein bands were visualized using ECL kit (Beyotime Institute of Biotechnology).

Dual-luciferase reporter assay. WT-PAX6-3'UTR or MUT-PAX6-3'UTR was inserted into the pmirGLO luciferase reporter vector (Promega Corporation). HCT-116 cells were transfected with the luciferase vector and miR-758 mimics using Lipofectamine $^{\circledR} 2000$ (Invitrogen; Thermo Fisher Scientifc, Inc.) according to the manufacturer's protocol. After $24 \mathrm{~h}$, the Renilla and firefly luciferase activity were detected using a dual-luciferase reporter assay system (Promega Corporation). Firefly luciferase activity was normalized to Renilla luciferase activity.

Statistical analysis. Data were analyzed using SPSS 17.0 (IBM Corp.) or GraphPad Prism 6 (GraphPad Software, Inc.) 
Table I. Relationship between miR-758 expression and clinicopathological characteristics of CRC patients.

\begin{tabular}{|c|c|c|c|c|}
\hline \multirow[b]{2}{*}{ Characteristics } & \multirow[b]{2}{*}{ Cases } & \multicolumn{2}{|c|}{$\operatorname{miR}-758$} & \multirow[b]{2}{*}{ P-value } \\
\hline & & High & Low & \\
\hline Age (years) & & & & 0.71 \\
\hline$\geq 60$ & 34 & 13 & 21 & \\
\hline$<60$ & 50 & 14 & 36 & \\
\hline Sex & & & & 0.53 \\
\hline Male & 32 & 14 & 18 & \\
\hline Female & 52 & 13 & 39 & \\
\hline Differentiation & & & & $0.01^{\mathrm{a}}$ \\
\hline Well & 58 & 16 & 42 & \\
\hline Moderate-poor & 26 & 11 & 15 & \\
\hline TNM stage & & & & $0.012^{\mathrm{a}}$ \\
\hline I-II & 64 & 18 & 46 & \\
\hline III-IV & 20 & 9 & 11 & \\
\hline Lymph node metastasis & & & & $0.02^{\mathrm{a}}$ \\
\hline No & 65 & 17 & 48 & \\
\hline Yes & 19 & 10 & 9 & \\
\hline
\end{tabular}

Statistical analyses were performed using $\chi^{2}$ test. ${ }^{a} \mathrm{P}<0.05$ was considered to indicate a statistically significant difference. CRC, colorectal cancer.
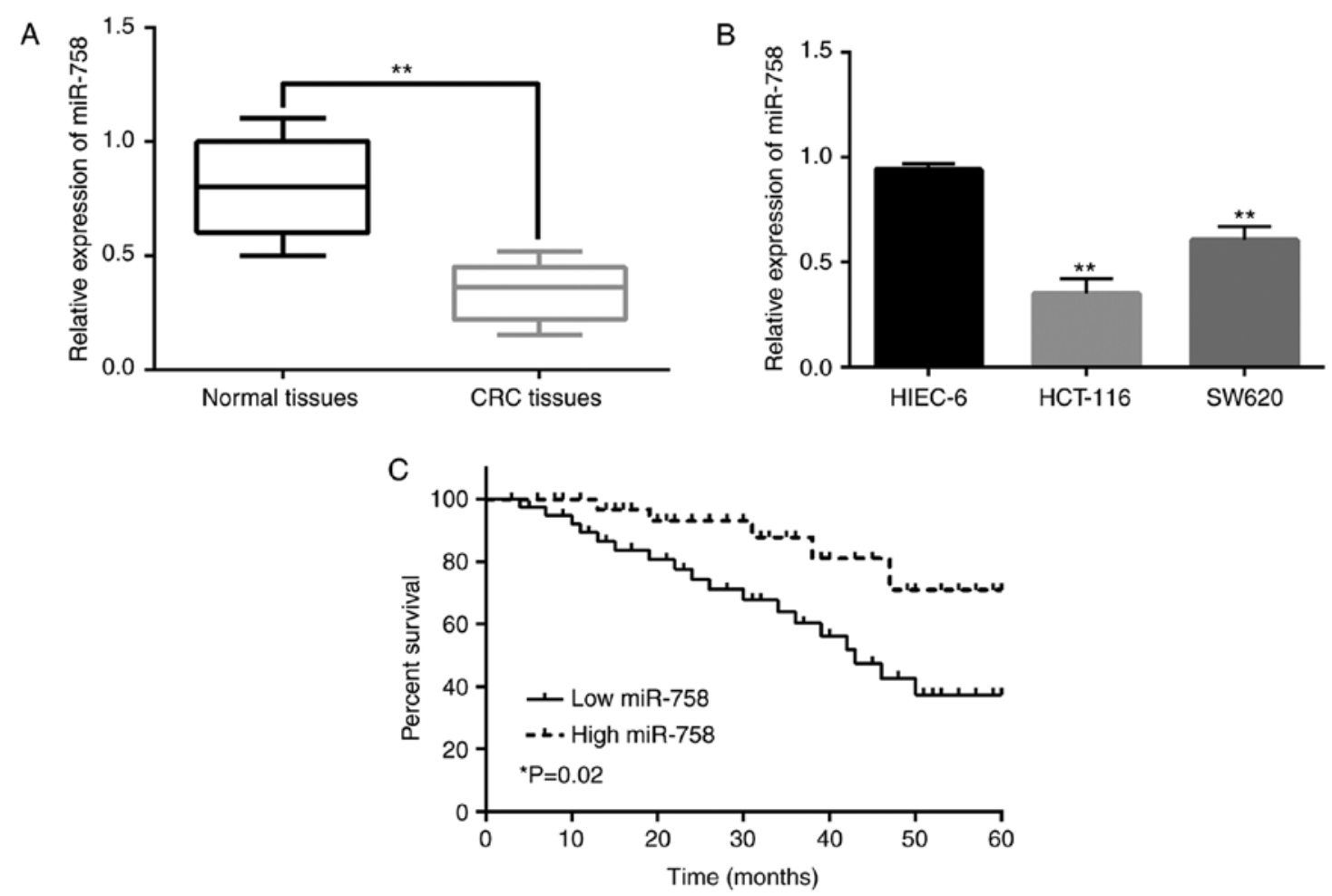

Figure 1. Downregulation of miR-758 is associated with aggressive behavior and poor prognosis in CRC. (A) miR-758 expression in normal and CRC tissues. (B) miR-758 expression levels in HCT-116, SW620 and HIEC-6 cells. (C) Low miR-758 expression was related to shorter overall survival in CRC patients. ${ }^{*} \mathrm{P}<0.05,{ }^{* *} \mathrm{P}<0.01$. CRC, colorectal cancer.

software and were expressed as the mean $\pm \mathrm{SD}$. The differences were analyzed using Student's t-test or one-way ANOVA followed by Tukey's post hoc test. The univariate Kaplan-Meier method with log-rank test and the Chi-square test were used to analyze the association between miR-758 and patient survival or clinical features. Spearman's rank correlation analysis was 

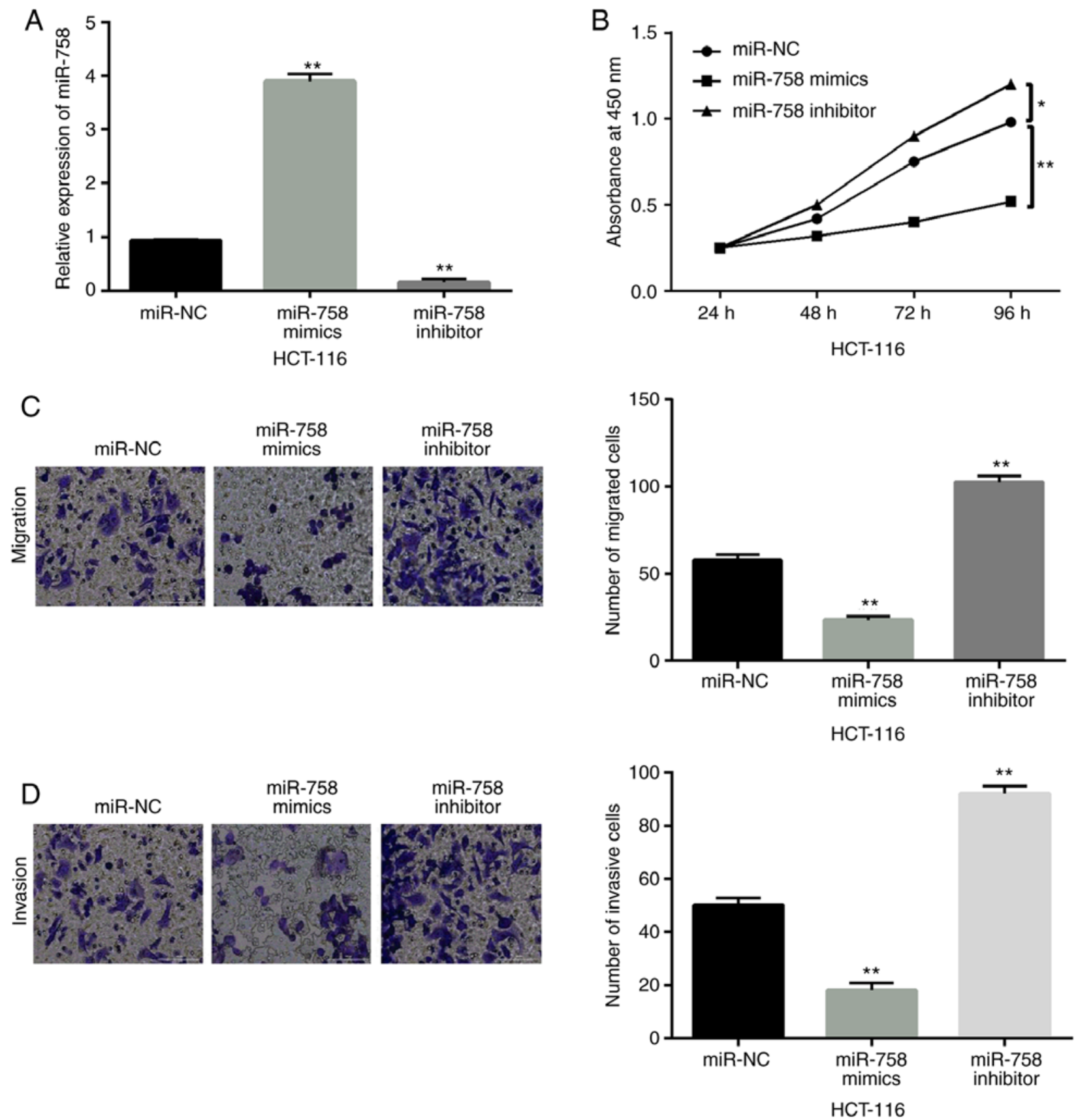

Figure 2. miR-758 restrains cell viability and metastasis in CRC. (A) miR-758 expression in HCT-116 cells with miR-758 mimics or inhibitor. (B-D) Cell proliferation, migration and invasion in HCT-116 cells with miR-758 mimics or inhibitor. ${ }^{*} \mathrm{P}<0.05,{ }^{* *} \mathrm{P}<0.01$. CRC, colorectal cancer.

performed to analyze the correlation between miR-758 and PAX6 expression levels. $\mathrm{P}<0.05$ was considered to indicate a statistically significant difference.

\section{Results}

Downregulation of miR-758 is associated with aggressive behavior and poor prognosis in CRC. RT-qPCR was performed to assess the expression of miR-758 in CRC. miR-758 expression was decreased in CRC tissues compared with that in normal tissues $(\mathrm{P}<0.01$, Fig. 1A). The downregulation of miR-758 was also detected in HCT-116 and SW620 cells in comparison with miR-758 expression in HIEC-6 cells $(\mathrm{P}<0.01$, Fig. 1B). Additionally, the low expression of miR-758 was found to be associated with differentiation, TNM stage and lymph-node metastasis in CRC patients $(\mathrm{P}<0.05$, Table I). Moreover, the poor prognosis in CRC patients was found to be associated with the downregulation of miR-758 ( $\mathrm{P}<0.05$, Fig. 1C). These results suggest that miR-758 may be involved in CRC progression. Since the difference of miR-758 expression between the HCT-116 and HIEC-6 cells was more significant than that between SW620 and HIEC-6 cells, HCT-116 cells were selected for the following experiments.

miR-758 restrains cell viability and metastasis in CRC. To investigate the function of miR-758 in CRC, a gain-loss function experiment was performed in HCT-116 cells with 

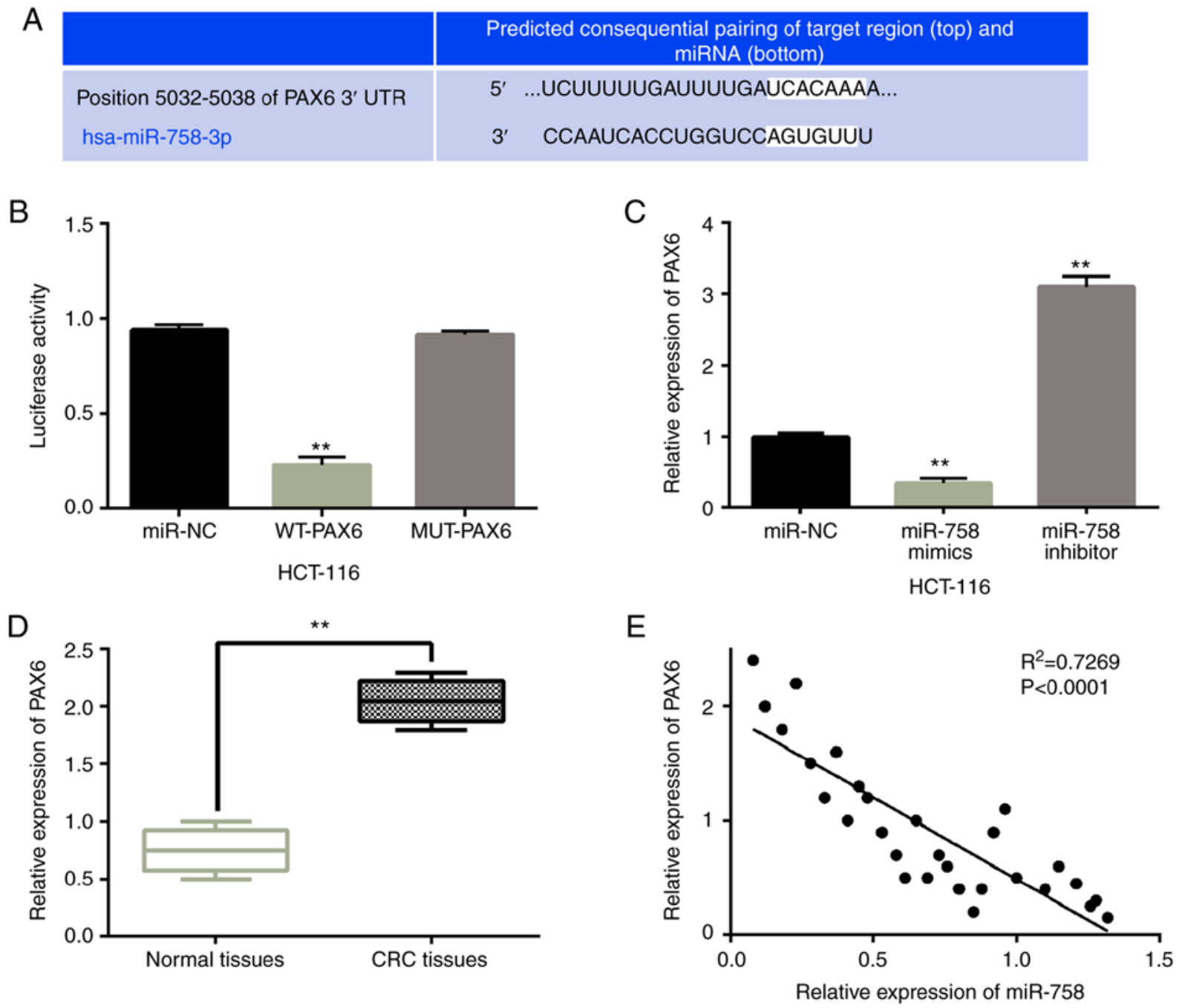

Figure 3. miR-758 directly targets PAX6. (A) Binding sites between miR-758 and PAX6. (B) Luciferase reporter assay. (C) PAX6 expression in HCT-116 cells with miR-758 mimics or inhibitor. (D) PAX6 expression in normal and CRC tissues. (E) Negative correlation of miR-758 expression with PAX6 expression. ${ }^{* *} \mathrm{P}<0.01$. PAX6, paired box 6; CRC, colorectal cancer.

miR-758 mimics or inhibitor. The expression of miR-758 was increased by its mimics and decreased by its inhibitor $(\mathrm{P}<0.01$, Fig. 2A). Functionally, the overexpression of miR-758 restrained cell proliferation $(\mathrm{P}<0.01)$, whereas the downregulation of miR-758 accelerated the proliferation of HCT-116 cells $(\mathrm{P}<0.05)$ (Fig. 2B). In addition, miR-758 mimics inhibited cell migration, whereas miR-758 inhibitor promoted cell migration in HCT-116 cells ( $\mathrm{P}<0.01$, Fig. 2C). Consistently, miR-758 mimics also inhibited cell invasion, whereas miR-758 inhibitor promoted HCT-116 cell invasion ( $\mathrm{P}<0.01$, Fig. 2D). Based on these results, miR-758 restrained CRC progression by inhibiting cell viability and metastasis.

miR-758 directly targets PAX6. For the prediction of the downstream target of miR-758, the TargetScan database was used (http://www.targetscan.org). It was predicted that miR-758 has a site that binds to the 3'-UTR of PAX6 (Fig. 3A). A luciferase reporter assay was then designed to confirm this prediction. miR-758 mimics were identified to reduce the luciferase activity of WT-PAX6 ( $<<0.01$, Fig. 3B). However, miR-758 mimics had no effect on MUT-PAX6 luciferase activity. To further confirm the relationship between miR-758 and PAX6, the PAX6 expression was observed in HCT-116 cells with miR-758 mimics or inhibitor. RT-qPCR showed that the overexpression of miR-758 reduced the expression of PAX6, whereas the downregulation of miR-758 promoted the expression of PAX6 ( $\mathrm{P}<0.01$, Fig. 3C). In addition, PAX6 was shown to be upregulated in CRC tissues compared with normal tissues $(\mathrm{P}<0.01$, Fig. 3D). Moreover, miR-758 was negatively correlated with PAX6 expression in CRC tissues $\left(\mathrm{P}<0.01, \mathrm{R}^{2}=0.7269\right.$; Fig. $\left.3 \mathrm{E}\right)$. The results suggest that miR-758 directly targets PAX6 and negatively regulates PAX6 expression in CRC.

miR-758 inhibits CRC progression through targeting PAX6. To explore the interaction between PAX6 and miR-758, PAX6 overexpression vectors were transfected into HCT-116 cells with miR-758 mimics. The results showed that the decreased expression of PAX6 induced by miR-758 mimics was restored by PAX6 overexpression vector in HCT-116 cells (Fig. 4A). Functionally, the overexpression of PAX6 attenuated the inhibitory effect of miR-758 on the cell proliferation of HCT-116 cells $(\mathrm{P}<0.01$, Fig. 4B). Consistently, miR-758-mediated inhibition of cell migration and invasion was also weakened by 
A

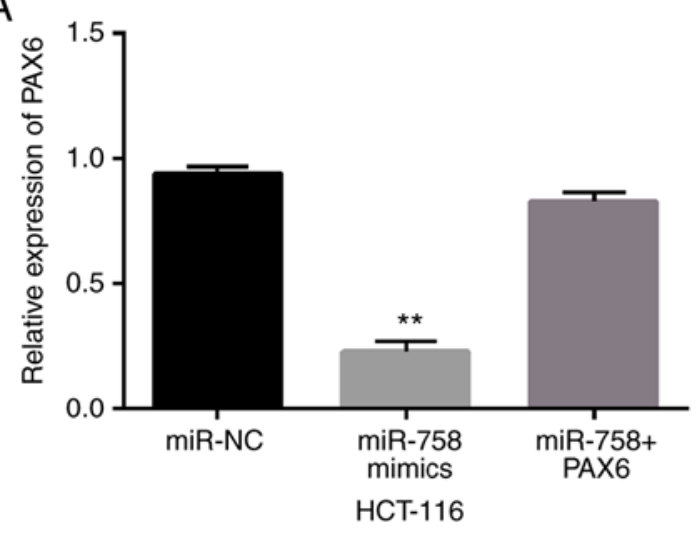

C
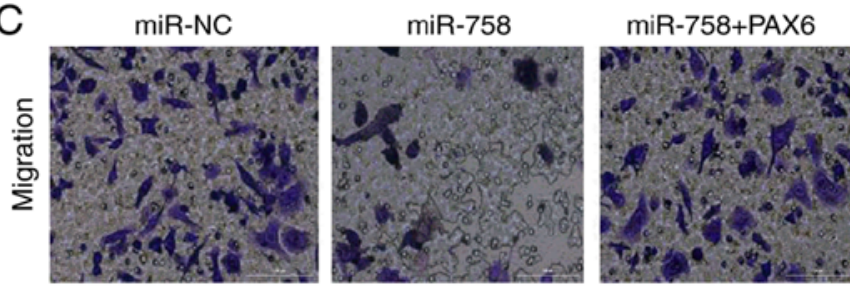

D

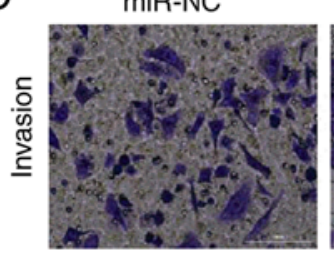

$\operatorname{miR}-758$

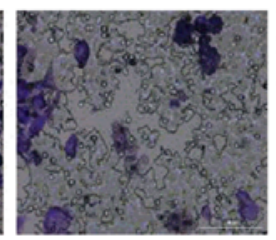

miR-758+PAX6

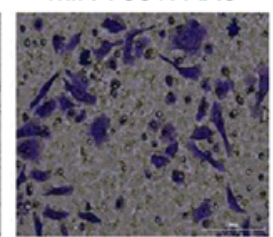

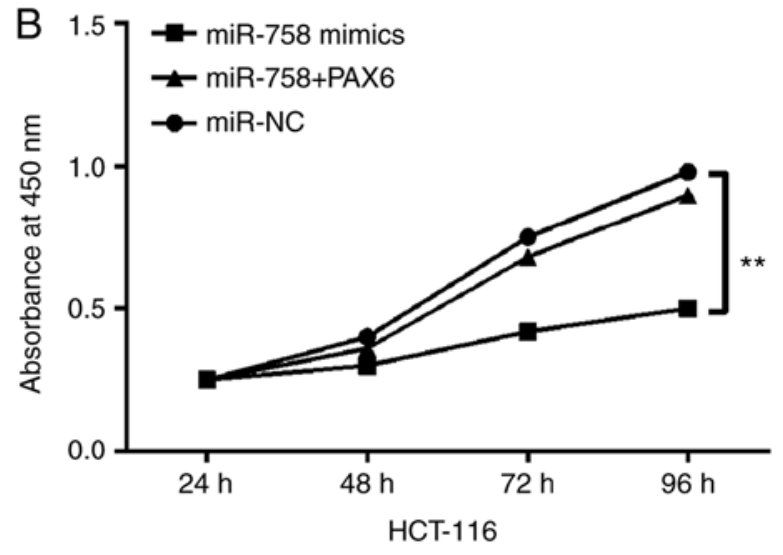

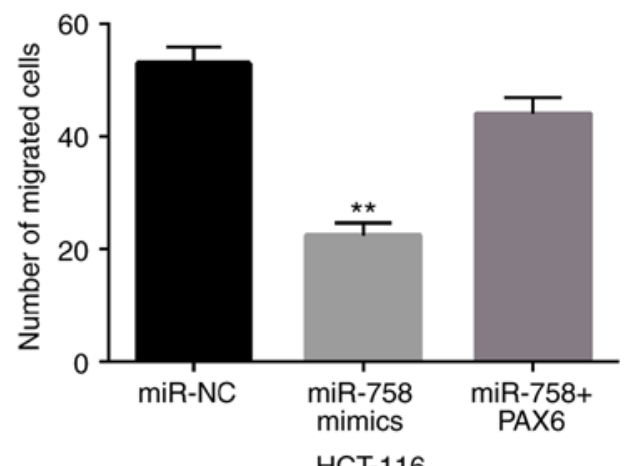

HCT-116

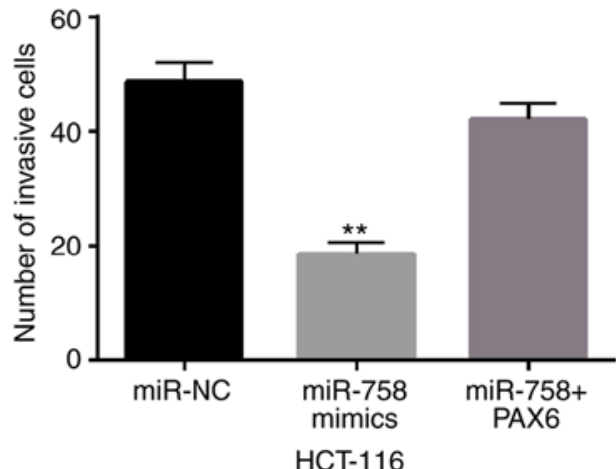

Figure 4. miR-758 inhibits CRC progression through targeting PAX6. (A) PAX6 expression in HCT-116 cells with PAX6 vector and miR-758 mimics. (B-D) Cell proliferation, migration and invasion in HCT-116 cells with PAX6 vector and miR-758 mimics ${ }^{* *} \mathrm{P}<0.01$. CRC, colorectal cancer; PAX6, paired box 6.

PAX6 overexpression ( $\mathrm{P}<0.01$, Fig. $4 \mathrm{C}$ and D). These results suggest that the upregulation of PAX6 impairs the inhibitory effect of miR-758 on CRC.

miR-758 blocks EMT and regulates PI3K/AKT pathway in $C R C$. To further illustrate the molecular mechanism of miR-758 in CRC, the way miR-758 regulates EMT (E-cadherin, $\mathrm{N}$-cadherin), apoptosis (Bcl-2, Bax) and PI3K/AKT pathway (PI3K, p-PI3K, AKT, p-AKT) in HCT-116 cells was investigated.Fig. 5 shows that the overexpression of miR-758 promoted E-cadherin and Bax expression levels, whereas miR-758 overexpression inhibited $\mathrm{N}$-cadherin and $\mathrm{Bcl}-2$ expression levels in HCT-116 cells. In addition, miR-758 mimics inhibited the expression of p-PI3K and p-AKT, whereas miR-758 inhibitor promoted their expression in HCT-116 cells (Fig. 5). However, miR-758 mimics did not affect the expression of PI3K and AKT (Fig. 5). Moreover, downregulation of miR-758 showed opposite effect on the expression of these genes (Fig. 5). Taken together, miR-758 overexpression blocked EMT and the $\mathrm{PI} 3 \mathrm{~K} / \mathrm{AKT}$ pathway in CRC.

\section{Discussion}

It has been shown that the abnormal expression of miRNAs is associated with CRC progression. For example, miR-769 has been reported to be downregulated in CRC and restrain cancer progression by targeting CDK1 (16). In the present study, the expression of miR-758 was also shown to be reduced in CRC. The downregulation of miR-758 was associated with aggressive behavior and poor prognosis in CRC patients. Functionally, miR-758 was presented to be able to inhibit cell viability and metastasis in CRC. Importantly, miR-758 was shown to block EMT and PI3K/AKT pathway in CRC, and induce apoptosis by regulating $\mathrm{Bcl}-2 / \mathrm{Bax}$ expression in $\mathrm{CRC}$. 


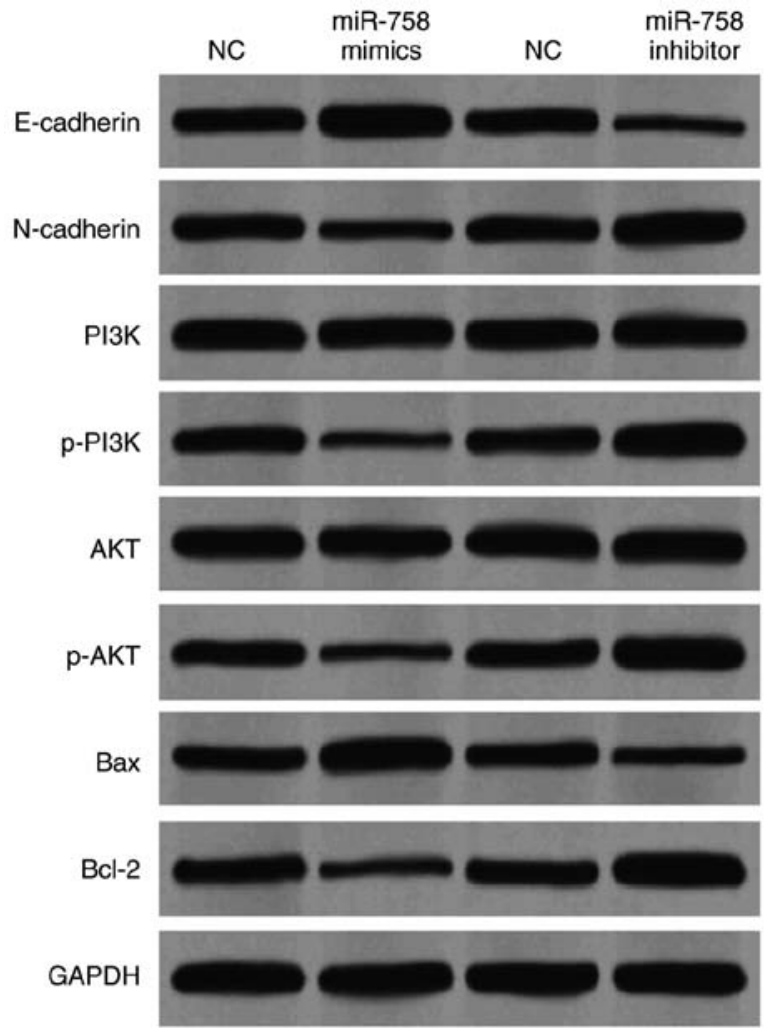

Figure 5. miR-758 blocks EMT and regulates PI3K/AKT pathway in CRC. The protein expression levels of E-cadherin, N-cadherin, PI3K, p-PI3K, AKT, p-AKT, Bax and Bcl-2 were regulated by miR-758 mimics or inhibitor in HCT-116 cells. CRC, colorectal cancer.

These results imply that miR-758 serves as a tumor suppressor in CRC progression.

Previous studies have also reported the downregulation of miR-758 in human cancers, such as ovarian and gastric cancer $(17,18)$. Also, miR-758 has been found to inhibit cell migration, invasion and proliferation in bladder cancer, hepatocellular carcinoma and glioblastoma (19-21). These results are consistent with the results of the present study. Downregulation of miR-758 has also been shown to predict poor prognosis in patients with non-small cell lung cancer (22) and the same result was also found in CRC patients. In addition, the present study showed that miR-758 induced apoptosis by suppressing Bcl-2 and enhancing Bax expression in CRC cells. Different from previous studies, EMT and PI3K/AKT pathway were also shown to be restrained by the overexpression of miR-758 in CRC cells. Up to our knowledge, these findings have not been reported by previous studies.

As previous studies have shown, miR-758 usually regulates tumorigenesis by mediating some target genes, such as SUCNR1 and CD36 $(23,24)$. In the present study, miR-758 was shown to directly target PAX6. Upregulation of PAX6 was detected in CRC tissues. More importantly, upregulation of PAX6 attenuated the inhibitory effect of miR-758 in CRC. In consistency with the results of the present study, PAX6 has also been reported to be upregulated in retinoblastoma and breast cancer $(25,26)$. In particular, as a target of miR-7, PAX6 was found to promote proliferation and invasion of CRC cells (27). These findings indicate that PAX6 acts as an oncogene in CRC. As a target gene, PAX6 has been regulated by several miRNAs including miR-335 and miR-365 $(28,29)$. In this research, a negative correlation between miR-758 and PAX6 was also found in CRC tissues. The interaction between miRNAs and PAX6 has also been investigated in other cancers. For example, miR-335 has been shown to attenuate the proliferation and invasion of breast cancer cells by regulating PAX6 (30). Wang et al demonstrated that miR-365b-3p blocked the cell cycle progression and promoted apoptosis in human retinoblastoma cells by downregulating PAX6 (31). Similarly, miR-758 restrained cell viability and metastasis in CRC by targeting PAX6, indicating that miR-758 is an inhibitory miRNA in CRC.

In conclusion, miR-758 was downregulated in CRC, which was associated with aggressive behavior and poor prognosis in CRC patients. Moreover, miR-758 restrained cell viability and metastasis in CRC via targeting PAX6 and was presented to block EMT and inactivate the PI3K/AKT pathway in CRC. The limitation of this study is that the role of miR-758 was only investigated in colon cancer cells HCT-116. The function of miR-758 in other subtypes of CRC cells is unclear. Further investigation of the specific regulatory mechanism of miR-758 in CRC will be the aim of our future research.

\section{Acknowledgements}

Not applicable.

\section{Funding}

No funding was received.

\section{Availability of data and materials}

The datasets used and/or analyzed during the present study are available from the corresponding author on reasonable request.

\section{Authors' contributions}

$\mathrm{XZ}$ was involved in the conception and design of the study. JX performed PCR, Transwell and CCK-8 assays. HZ was responsible for western blot analysis and dual-luciferase reporter assay. JS analyzed and interpreted the patient data. $\mathrm{NL}$ and XH assisted with statistical analysis. All authors read and approved the final manuscript.

\section{Ethics approval and consent to participate}

The study was approved by the Institutional Ethics Committee of Qingdao West Coast New Area Central Hospital (Qingdao, China). Patients who participated in this research had complete clinical data. Signed written informed consents were obtained from the patients and/or guardians.

\section{Patient consent for publication}

Not applicable.

\section{Competing interests}

The authors declare that they have no competing interests. 


\section{References}

1. Bray F, Ferlay J, Soerjomataram I, Siegel RL, Torre LA and Jemal A: Global cancer statistics 2018: GLOBOCAN estimates of incidence and mortality worldwide for 36 cancers in 185 countries. CA Cancer J Clin 68: 394-424, 2018.

2. Primavesi F, Stattner S, Jager T, Gobel G, Presl J, Tomanova K, Buchner S, Maglione M, Resch T, Hutter J, et al: Progressive oncological surgery is associated with increased curative resection rates and improved survival in metastatic colorectal cancer. Cancers (Basel) 11: E218, 2019.

3. Kopetz S, Chang GJ, Overman MJ,Eng C, Sargent DJ,Larson DW, Grothey A, Vauthey JN, Nagorney DM and McWilliams RR Improved survival in metastatic colorectal cancer is associated with adoption of hepatic resection and improved chemotherapy. J Clin Oncol 27: 3677-3683, 2009.

4. Bartel DP: MicroRNAs: Genomics, biogenesis, mechanism, and function. Cell 116: 281-297, 2004.

5. Liang Z, Li X, Liu S, Li C, Wang X and Xing J: MiR-141-3p inhibits cell proliferation, migration and invasion by targeting TRAF5 in colorectal cancer. Biochem Biophys Res Commun 514: 699-705, 2019.

6. Li X, Zhang X, Zhang Q and Lin R: miR-182 contributes to cell proliferation, invasion and tumor growth in colorectal cancer by targeting DAB2IP. Int J Biochem Cell Biol 111: 27-36, 2019.

7. Zhou GH, Lu YY, Xie JL, Gao ZK, Wu XB, Yao WS and Gu WG: Overexpression of miR-758 inhibited proliferation, migration, invasion, and promoted apoptosis of non-small cell lung cancer cells by negatively regulating HMGB. Biosci Rep 39: BSR20180855, 2019.

8. Meng X, Zhao Y, Wang J, Gao Z, Geng Q and Liu X: Regulatory roles of miRNA-758 and matrix extracellular phosphoglycoprotein in cervical cancer. Exp Ther Med 14: 2789-2794, 2017.

9. Elso C, Lu X, Weisner PA, Thompson HL, Skinner A, Carver E and Stubbs L: A reciprocal translocation dissects roles of Pax6 alternative promoters and upstream regulatory elements in the development of pancreas, brain, and eye. Genesis 51: 630-646, 2013.

10. Li L, Li B, Zhang H, Bai S, Wang Y, Zhao B and Jonas JB: Lentiviral vector-mediated PAX6 overexpression promotes growth and inhibits apoptosis of human retinoblastoma cells. Invest Ophthalmol Vis Sci 52: 8393-8400, 2011.

11. Zong X, Yang H, Yu Y, Zou D, Ling Z, He X and Meng X: Possible role of Pax-6 in promoting breast cancer cell proliferation and tumorigenesis. BMB Rep 44: 595-600, 2011.

12. Needhamsen M, White RB, Giles KM, Dunlop SA and Thomas MG: Regulation of Human PAX6 Expression by miR-7. Evol Bioinform Online 10: 107-113, 2014.

13. Slattery ML, Mullany LE, Sakoda LC, Wolff RK, Stevens JR, Samowitz WS and Herrick JS: The PI3K/AKT signaling pathway: Associations of miRNAs with dysregulated gene expression in colorectal cancer. Mol Carcinog 57: 243-261, 2018.

14. Song Y,Zhao Y, Ding X and Wang X: microRNA-532 suppresses the PI3K/Akt signaling pathway to inhibit colorectal cancer progression by directly targeting IGF-1R. Am J Cancer Res 8: 435-449, 2018.

15. Livak KJ and Schmittgen TD: Analysis of relative gene expression data using real-time quantitative PCR and the 2(-Delta Delta C(T)) method. Methods 25: 402-408, 2001.

16. Wang L, Xu M, Lu P and Zhou F: microRNA-769 is downregulated in colorectal cancer and inhibits cancer progression by directly targeting cyclin-dependent kinase 1 . Onco Targets Ther 11: 9013-9025, 2018.
17. Hu X, Li Y, Kong D, Hu L, Liu D and Wu J: Long noncoding RNA CASC9 promotes LIN7A expression via miR-758-3p to facilitate the malignancy of ovarian cancer. J Cell Physiol 234: 10800-10808, 2019.

18. Guo J, Zhang Z, Pan L and Zhou Y: Identification of miR-758-3p as potential modulator of CBX5 expression in gastric cancer. Technol Cancer Res Treat 17: 1533033818816061, 2018.

19. Wu X, Chen B, Shi H, Zhou J, Zhou F, Cao J and Sun X: miR-758-3p suppresses human bladder cancer cell proliferation, migration and invasion by targeting NOTCH2. Exp Ther Med 17: 4273-4278, 2019.

20. Jiang D, Cho W, Li Z, Xu X, Qu Y, Jiang Z, Guo L and Xu G: MiR-758-3p suppresses proliferation, migration and invasion of hepatocellular carcinoma cells via targeting MDM2 and mTOR. Biomed Pharmacother 96: 535-544, 2017.

21. Liu J, Jiang J, Hui X, Wang W, Fang D and Ding L: MiR-758-5p suppresses glioblastoma proliferation, migration and invasion by targeting ZBTB20. Cell Physiol Biochem 48: 2074-2083, 2018.

22. Wang S and Jiang M: The long non-coding RNA-DANCR exerts oncogenic functions in non-small cell lung cancer via miR-758-3p. Biomed Pharmacother 103: 94-100, 2018.

23. Zhang H, Zheng J, Lin J, Chen J, Yu Z, Chen C and Liu T: miR-758 mediates oxLDL-dependent vascular endothelial cell damage by suppressing the succinate receptor SUCNR1. Gene 663: 1-8, 2018.

24. Li BR, Xia LQ, Liu J, Liao LL, Zhang Y, Deng M, Zhong HJ, Feng TT, He PP and Ouyang XP: miR-758-5p regulates cholesterol uptake via targeting the CD36 3'UTR. Biochem Biophys Res Commun 494: 384-389, 2017.

25. Li X, Yang L, Shuai T, Piao T and Wang R: MiR-433 inhibits retinoblastoma malignancy by suppressing Notch1 and PAX6 expression. Biomed Pharmacother 82: 247-255, 2016.

26. Zou Q, Yi W, Huang J, Fu F, Chen G and Zhong D: MicroRNA-375 targets PAX6 and inhibits the viability, migration and invasion of human breast cancer MCF-7 cells. Exp Ther Med 14: 1198-1204, 2017.

27. Li Y, Li Y, Liu Y, Xie P, Li F and Li G: PAX6, a novel target of microRNA-7, promotes cellular proliferation and invasion in human colorectal cancer cells. Dig Dis Sci 59: 598-606, 2014.

28. Cheng Q, Cao H, Chen Z, Ma Z, Wan X, Peng R and Jiang B: PAX6, a novel target of miR-335, inhibits cell proliferation and invasion in glioma cells. Mol Med Rep 10: 399-404, 2014.

29. Yuan F, Liu J, Pang H, Tian Y, Yuan K, Li Y, Wang J, Bian S, Zheng Y, Dong D, et al: MicroRNA-365 suppressed cell proliferation and migration via targeting PAX6 in glioblastoma. Am J Transl Res 11: 361-369, 2019.

30. Meng Y,Zou Q, Liu T, Cai X, Huang Y and Pan J: microRNA-335 inhibits proliferation, cell-cycle progression, colony formation, and invasion via targeting PAX6 in breast cancer cells. Mol Med Rep 11: 379-385, 2015.

31. Wang J, Wang X, Wu G, Hou D and Hu Q: MiR-365b-3p, down-regulated in retinoblastoma, regulates cell cycle progression and apoptosis of human retinoblastoma cells by targeting PAX6. FEBS Lett 587: 1779-1186, 2013.

(i) (5) $\ominus$ This work is licensed under a Creative Commons Attribution-NonCommercial-NoDerivatives 4.0 International (CC BY-NC-ND 4.0) License. 